\title{
Éditorial: Présentation du contexte, des pratiques et des politiques en matière de soins d'une population vieillissante
}

La génération des baby-boomers vieillit lentement mais sûrement; ses années d'activité passant pour se rapprocher de ses vieux jours. Les implications pour la santé et les soins sociaux sont à l'avant-garde de la sensibilisation du public et du débat politique, et l'on s'inquiète de la capacité des personnes âgées et des gouvernements à financer la retraite des baby-boomers. Les gérontologues ont joué et continuent de jouer un rôle important dans la préparation à un vieillissement de la population en fournissant la preuve par leur recherche qu'un changement est nécessaire. En particulier, comme ce numéro spécial le montrera, les gérontologues s'appliquent à (1) décrire l'expérience actuelle des adultes d'âge moyen et avancé (le contexte), (2) développer et évaluer de nouveaux modèles de soins (la pratique), (3) faire des projections sur l'avenir du vieillissement de la population en se basant sur les tendances actuelles et (4) préconiser des solutions politiques pour améliorer les soins de la population vieillissante (la politique).

Ce numéro spécial de La Revue canadienne du vieillissement présente les résultats de la recherche concernant les resultats de prendre soin d'un vieillissement de la population par les chercheurs affiliés avec le programme de recherche pluridisciplinaire, sociales et économiques des dimensions d'une population de vieillissement (SEDAP-II). Le financement majeur pour SEDAP-II a été fourni par le Conseil de recherches en science humaines du Canada (CRSH) aux termes de la concurrence des Grands Travaux de Recherche concertée (GTRC). Elle implique 46 institutions de 14 universités canadiennes et de 3 universités étrangères. ${ }^{1}$

SEDAP-II étudie comment le vieillissement de la population affectera la main-d'œuvre et l'économie. Les articles présentés dans ce numéro spécial peuvent être divisés grossièrement en quatre sections: (1) quatre articles abordent les besoins futurs de la population vieillissante en décrivant le contexte actuel de vieillissement et en faisant des projections pour le futur; (2) deux articles abordent le contexte actuel des deux groupes différents de soignants; (3) deux articles abordent la relation entre les prestataires de soins à domicile et les bénéficiaires de soins; et (4) deux articles décrivent et évaluent l'effort d'intervention en cas de chute chez un groupe de personnes âgées à risque qui reçoivent des services de soins à domicile.

\section{Réponse aux besoins futurs d'une population vieillissante}

Préserver leur indépendance est un objectif que les aînés ont classé comme partie intégrante de leur qualité de vie. Alors que la plupart des aînés vivant chezsoi se déclarent en bonne santé, la grande majorité rapporte au moins une maladie chronique diagnostiquée par un professionnel de la santé. Certaines maladies chroniques sont plus susceptibles que les autres de mener à l'invalidité, et le type de handicap expérimenté est lié à la maladie. Un handicap qui limite les activités de la vie quotidienne peut avoir un impact profond sur la vie des personnes âgées, y compris sur leur mobilité, leur agilité, la possibilité pour eux d'accomplir les activités de la vie quotidienne, leurs activités de loisirs et leur bien-être psychologique global. On craint qu'avec le vieillissement de la population canadienne, le nombre de personnes atteintes de maladies chroniques augmentera considérablement, ce qui entraîne une augmentation des coûts du système de soins de santé et davantage de contraintes pour le système de soins à domicile et d'aide sociale ainsi que pour les soignants qui aident ces personnes âgées de manière informelle.

Dans le premier article qui traite des besoins futurs d'une population vieillissante, « Maladies chroniques: modification de la prévalence chez une population vieillissante et implications pour la prestation des services de santé ", Frank Denton et Byron Spencer montrent comment la prévalence chez la population générale d'un ensemble de maladies chroniques augmentera considérablement au cours du prochain quart de siècle en raison des changements de projection de la répartition par âge prévus. Si le nombre de maladies devait être maintenu, la projection des auteurs indique que les exigences en matière de santé augmenteraient plus rapidement que la population plus de deux fois plus rapidement dans le cas des séjours hospitaliers. Toutefois, Denton et Spencer démontrent que, s'il y avait une hypothétique réduction du nombre de maladies chroniques par habitant, cela aurait un impact significatif sur l'utilisation des ressources de santé et que cela entraînerait des économies substantielles. Leurs résultats ont des implications politiques et pratiques pour promouvoir un vieillissement en bonne santé. 
Des variations régionales importantes existent quant à la disponibilité des services de santé et au financement parmi les communautés rurales. Bien sûr, les services publics subventionnés et privés ont généralement été caractérisés comme "limités » dans les zones rurales, comparativement aux centres urbains. En partie, ce peut être attribué à la méthode d'affectation des fonds aux soins de santé et de soins à domicile - typiquement selon la taille de la population - limitant souvent dramatiquement les ressources financières des petites collectivités fréquemment, sans compter que ces dernières sont souvent en manque de professionnels de la santé. En outre, les communautés rurales sont généralement plus âgées que leurs homologues urbains.

Le deuxième article sur les besoins futurs d'une population vieillissante s'intitule " La géographie est-elle importante? Usage des services de santé et besoins non- satisfaits des Canadiens âgés. " Dans celui-ci, James Ted McDonald et Heather Conde examinent, par le biais de l'enquête sur les indicateurs de santé communautaire canadienne 2002-03, si l'usage des services de santé de base et si l'incidence des besoins nonsatisfaits en matière de soins de santé par les Canadiens âgés varient entre les zones urbaines et rurales du pays. Les auteurs essaient de déterminer si les habitants des régions rurales sont susceptibles de rencontrer davantage d'obstacles à l'obtention de soins de santé que leurs homologues urbains. D'une part, ils ont trouvé que le nombre de mesures importantes concernant l'usage des services de santé (c'est-à-dire, les visites chez un généraliste, un spécialiste et un dentiste) est inférieur pour les Canadiens âgés qui habitent dans les zones rurales comparé à ceux qui habitent dans les zones urbaines. D'autre part, ils n'ont trouvé aucune différence en ce qui concerne les nuits à l'hôpital ou les besoins non-satisfaits en soins de santé, un résultat qui suggère que, pour les résidants ruraux, la perception des besoins peut-être être liée au manque de services de soins de santé dans leur région. En termes de politique et de pratique, les résultats des auteurs soulignent davantage les besoins en services de soins de santé dans les zones rurales et la nécessité de mettre en place des initiatives politiques pour insister sur l'importance de l'accès rapide aux soins de santé et de la médecine préventive pour les habitants des régions rurales.

Nous avons effectué des recherches de base sur le bienêtre financier des aînés retraités au Canada. Nous connaissons la distribution des revenus des retraités, leurs sources de revenu, et nous avons identifié les groupes de personnes âgées vivant au niveau du seuil de pauvreté et en-deçà de celui-ci. Une question qui a été débattue par les décideurs politiques et les conseillers en planification financière est la suivante: « de combien les aînés ont-ils besoin pour financer leur retraite? » Dans leur article abordant le thème des besoins d'une population vieillissante intitulé "Coûts des besoins de base pour les personnes âgées canadiennes », Bonnie Jeanne MacDonald, Doug Andrews et Robert Brown ont déterminé le montant de revenu net après impôt qui serait nécessaire pour financer les besoins fondamentaux, y compris les soins de santé, pour les aînés canadiens vivant dans différentes circonstances dans cinq grandes villes canadiennes. Leur étude établit que les frais de base varient énormément selon les circonstances de la vie des personnes âgées, et que le coût de la vie global d'une personne âgée est proche ou même supérieur à celui d'un adulte vivant dans des circonstances similaires. Ceci va à l'encontre de la conclusion plus souvent admise que les personnes âgées ont besoin de moins de revenus que leurs homologues qui sont encore dans la vie active. Les auteurs soutiennent en outre que les prestations maximales cumulées de la Sécurité vieillesse (SV) et du Supplément de revenu garanti (SRG) ne sont pas suffisantes pour satisfaire le coût des besoins essentiels pour les personnes âgées à faible revenu qui louent leur logement et qui comptent sur les transports publics. Ils concluent que les circonstances individuelles, plutôt que le statut de retraite per se, sont les éléments principaux dans le calcul du coût des besoins essentiels. Les auteurs recommandent qu'en termes de politique et de pratique, les aînés et les planificateurs financiers ne se fient pas aveuglément au niveau de revenu universel ou au ratio de remplacement du revenu fixe pour faire leur projection du niveau de revenu nécessaire pour être en mesure de prendre sa retraite.

Le pourcentage des personnes âgées au Canada est censé augmenter considérablement au cours des deux prochaines décennies. Sur le nombre de personnes âgées recevant des soins, environ la moitié reçoivent tous leurs soins de leur famille et amis, et il y a eu un glissement vers l'augmentation de la dépendance des soignants appartenant à la famille, loin des institutions et du système de soins de santé formel. Dans le dernier article abordant les besoins d'une population vieillissante, "Quelle sera la composition de la famille des plus âgés demain? Étude comparative du Canada et de la France », Joëlle Gaymu et ses collaborateurs soutiennent que les comparaisons avec la France sont intéressantes parce que la société française a eu une longueur d'avance en termes de vieillissement de la population, et que, d'ici moins de 30 ans, la proportion des personnes âgées au Canada sera semblable à celle de la France. Le Canada peut regarder la France et les autres pays européens afin d'évaluer leurs réponses politiques aux défis de la prise en charge du vieillissement de la population.

À l'aide du modèle de micro simulation longitudinale LifePaths, les auteurs montrent comment les 
changements de structure de la pyramide des âges, la proportion des sexes, le statut matrimonial, et la proportion de personnes sans enfant survivant transformera le réseau familial des personnes âgées à la fois en termes de structure et de nombre au Canada et en France. Bien qu'ils notent des différences entres les pays, ils démontrent que, d'une part, au cours des vingt-cinq prochaines années, le nombre de parents potentiellement susceptibles d'aider leurs aînés (principalement les conjoints et enfants) augmentera du fait de l'effet de la génération baby-booms. D'autre part, la population plus dépendante de soins formels, sans aucun soutien potentiel d'un enfant ou d'un conjoint, augmentera également, et la pénurie des soignants de la parenté est susceptible de devenir plus aiguë après 2030, en raison de différents changements dans la composition familiale. Selon les auteurs, les politiques et les programmes dans les deux pays devront se préparer au fait qu'un plus grand nombre de conjoints âgés seront les soignants et, qu'au Canada, il faudra se préoccuper de l'augmentation significative du nombre de personnes qui s'appuieront sur des services formels.

\section{Divers groupes de soignants}

Des recherches approfondies sur les soins décrivent les aidants, évaluant leur contribution tant sur le plan des économies qu'ils font faire au système de soins de santé et sur le plan de la qualité des soins qui sont fournis. Ces recherches examinent aussi la santé des aidants et leur besoin de répit. L'un des aspects étudiés de plus près dans ces recherches concerne le niveau de contraintes et la charge auxquels font face les soignants, bien que d'autres recherches mentionnent un facteur de "gratification» associé à ces soins.

La deuxième série d'articles concrétise les expériences, les perceptions et les besoins de deux groupes distincts de soignants. Lori Campbell décrit les expériences des fils qui dispensent des soins à un parent âgé dans l'article intitulé "Ces fils attentifs: exploration de l'expérience masculine dans l'aide filiale. » Elle cherche à identifier dans quelle mesure leur compréhension de la prestation diffère selon le statut matrimonial et la cohabitation.

Son analyse est organisée autour de six thèmes, dont: (1) «Parce que c'est la famille »; (2) « Proche ou plus proche avec les soins »; (3) «Un mélange d'émotions »; (4) « Prestation de soins, un processus progressif »; (5) "Identité en tant que fils soignant: croissance personnelle », et (6) " changements effectués par le biais de soins. » Malgré les différences manifestées par leur contexte social, elle montre que les fils ont un engagement ferme et un vrai souci de leurs parents. Elle décrit, en particulier, l'engagement central que prennent les fils jamais mariés qui résident chez leurs parents - dont la majorité n'a pas travaillé à temps plein - pour s'occuper de leur parent et les implications spécifiques que cette expérience de soins a sur leur sécurité financière future.

Dans leur article intitulé "L'arbre généalogique: congruence de l'obligation filiale entre les parents âgés et les enfants adultes dans les familles canadiennesjaponaises ", Karen Kobayashi et Laura Funk explorent les différences intergénérationnelles en ce qui concerne cette obligation filiale parmi les parents les plus âgés et les enfants adultes dans les familles canadiennesjaponaises issues de l'immigration. Ils utilisent leurs entretiens avec 100 dyades parent-enfant en ColombieBritannique pour explorer l'hypothèse de jeu intergénérationnel désormais classique, qui maintient que les parents et les enfants ont des attentes et des perceptions différentes de la relation filiale en raison de différences du développement dans leurs préoccupations ou « enjeux ». L'analyse des auteurs conduit à contester cette hypothèse car la majorité des dyades parent-enfant suggère un accord global dans cette obligation filiale quels que soient les différents contextes sociaux (sexe du parent, état matrimonial, et état de santé du parent). Leur étude pointe vers la forme de solidarité durable dans les familles canadiennes-japonaises, mais ils préviennent que les gouvernements ne devraient pas utiliser l'hypothèse de la solidarité familiale et de la prise en charge comme excuse pour se décharger de leurs responsabilités en matière de soins des aînés sur les familles.

\section{Les soignants et les prestataires de soins à domicile}

Les soins à domicile sont le segment qui connaît la croissance la plus rapide dans le secteur de la santé au Canada. Le troisième ensemble $d$ 'articles examine les problèmes rencontrés par les prestataires d'aide à domicile et nous rapporte des expériences de soignants formels. Dans leur article intitulé «Stratégies utilisées par les prestataires d'aide à domicile dans le service aux personnes âgées ", Joanie SimsGould et Anne Martin-Matthews utilisent les données d'entretiens avec 118 prestataires de services d'aide domicile en Colombie-Britannique pour examiner un modèle conceptuel qui localise les prestataires de services d'aide à domicile, le client âgé et le membre de la famille à l'intersection des sphères publiques et privées encadrées par leurs caractéristiques sociales, spatiales, temporelles, et la structure organisationnelle de leur entreprise. Leur analyse informe et approfondit notre compréhension de l'expérience professionnelle des prestataires de services d'aide à domicile. 
Malgré le fait que le secteur des soins de santé canadien dépende de plus en plus de travailleurs immigrés pour les soins à domicile et les soins de longue durée au Canada, on connaît très peu de choses sur les expériences des travailleurs et leurs relations avec les clients. L'article d'Ivy Lynn Bourgeault et collaborateurs intitulé «Relations entre prestataires de soins immigrés et personnes âgées en soins de longue durée» vise à examiner les relations des prestataires de soins immigrés avec leurs clients aînés. L'analyse des auteurs révèle des aperçus importants au regard des différences raciales ou ethniques, des différences culturelles et des barrières linguistiques entre les prestataires de soins à domicile et leurs clients et comment celles-ci compliquent leurs relations. Cela est encore aggravé par la pénurie de personnel, des charges de travail élevées et la négligence générale des soins des aînés. L'article se termine par une discussion sur les implications politiques, notamment sur la nécessité de programmes éducatifs spéciaux, culturellement sensibles pour les prestataires de soins immigrés et les familles et soignants, et sur la nécessité d'envisager autant que possible un contexte culturel commun entre le prestataire de soins immigré et l'aîné auquel il apporte les soins.

\section{L'Intervention en cas de chute}

Le Canada est un chef de file en médecine factuelle et nous mettons au point des lignes directrices sur les pratiques exemplaires dans la prestation des soins aux personnes âgées. En conséquence, nous allons terminer ce supplément spécial avec deux articles de Maureen Markle-Reid et collaborateurs qui présentent les résultats d'une approche d'équipe multifactorielle et multidisciplinaire à la prévention des chutes pour les aînés qui sont pris en charge à domicile. Le premier article, intitulé " Étude transversale de l'importance, des corrélations, et du coût des chutes des clients aînés à risque de chute qui sont pris en charge à domicile ", rapporte les résultats à la base d'un essai contrôlé aléatoire sur 109 personnes âgées de 75 ans et plus, dont 71 pour cent avait signalé une chute au cours des six mois précédents. Les contributions majeures de l'étude sont qu'elle se concentre sur les chutes et chutes évitées de peu (glissades et trébuchements), et qu'elle évalue l'usage et les coûts de la gamme complète des services de santé. Le rapport de l'étude révèle le caractère multifactoriel des chutes et identifie plusieurs facteurs de risque interactifs, dont certains sont modifiables. Le deuxième article, intitulé « Effets et coûts d'une équipe multidisciplinaire et d'une approche multifactorielle de la prévention des chutes pour des clients âgés pris en charge à domicile et à risque pour les chutes: essai aléatoire contrôlé » expose les résultats d'une intervention -une stratégie de prévention multifactorielle et fondée sur des données factuelles sur six mois impli- quant une équipe multidisciplinaire. Les résultats de l'étude fournissent la preuve que la présélection, suivie par des interventions ciblées visant plusieurs facteurs et menées par une équipe multidisciplinaire, entraîne des améliorations de la qualité de vie liées à la santé, la réduction des chutes pour certains types de personnes âgées et une réduction du nombre de glissades et de trébuchements, sans aucun frais de soins de santé supplémentaires. Les résultats de l'étude confirment les positions de la littérature sur les directives de pratique exemplaire pour assurer la prévention des chutes chez les personnes âgées à risques et concluent avec les recommandations pour les décideurs politiques de soins à domicile, les agences et les bailleurs de fonds pour assurer une approche multidisciplinaire de la prévention des chutes.

\section{Conclusion}

Une grande partie de la politique publique au Canada s'inquiète des conséquences du vieillissement de la population. La nécessité de changement des politiques et des programmes pour faire face aux projections de l'augmentation du nombre et de la proportion des personnes âgées est désormais admise. La recherche jouera un rôle important en décrivant l'expérience actuelle des adultes d'âge moyen et des aînés (le contexte), en développant et en évaluant de nouveaux modèles de soins (la pratique), en faisant des projections sur l'avenir de la population vieillissante basées sur les tendances actuelles, et en préconisant des solutions politiques pour améliorer les soins d'une population vieillissante (la stratégie). Les articles de recherche présentés ici fournissent des informations et des suggestions pour aider les décideurs politiques et les planificateurs à préparer la voie à suivre, mais il faudra rassembler beaucoup d'informations complémentaires si l'on veut répondre aux besoins de notre population vieillissante.

Le système de soins à long terme du Canada sera considérablement modifié à l'avenir pour fournir une meilleure qualité de soins aux aînés dans leur propre foyer et dans les maisons de soins infirmiers. Il est admis que les baby-boomers sont une génération très différente de leurs parents et qu'ils exigeront des modifications aux soins de santé individuels et communautaires. Ils voudront vieillir chez-soi et être aidés par des prestations de soins individuelles et communautaires. En outre, s'ils ne sont plus capables de vivre de façon indépendante ou s'ils souhaitent changer de résidence, ils exigeront un logement alternatif abordable pour répondre à leurs besoins. Ils s'attendront à ce que les maisons de soins infirmiers fournissent des soins de qualité, y compris des activités récréatives stimulantes, une bonne nourriture, et 
des possibilités d'exercice physique et de réhabilitation. Si cette hypothèse est vraie, nous demandons, pourquoi les baby-boomers ne sont pas plus loquaces pour exiger des modifications du système de soins pour leurs parents, dont certains reçoivent des soins actuellement ou auront besoin de soins dans un très proche avenir. Après tout, lorsque les baby-boomers atteindront le point dans leur vie où ils auront besoin de services pour maintenir leur indépendance, il sera peut-être trop tard pour que les modifications nécessaires pour répondre à leurs besoins entrent en vigueur. C'est maintenant qu'il faut agir! En tant que chercheurs et enseignants, nous avons la responsabilité morale de déployer nos connaissances et notre expertise pour renseigner les pratiques et le débat politique en vue d'améliorer la qualité des soins et la qualité de vie de notre population aînée.

Margaret A. Denton \& Isik U. Zeytinoglu

Rédacteurs invités

\section{Note}

1 Le soutien financier supplémentaire a été assurée par les universités suivantes: McMaster, Toronto, Queen's, Waterloo, York, Carleton, Memorial, Calgary et Victoria. Les institutions suivantes ont également apporté un soutien financier: Statistique Canada, Développement social Canada, Citoyenneté et Immigration Canada, SFI (Institut national de recherche sociale au Danemark), IZA (Institut pour l'étude de la main-d'œuvre en Allemagne) et ICIS (Institut canadien d'information sur la santé). 